\title{
Effect of stress on the leaf anatomy of sugarcane cultivars with different drought tolerance (Saccharum officinarum, Poaceae)
}

\author{
Worasitikulya Taratima ${ }^{1 *}$, Thapakorn Ritmaha ${ }^{1}$, Nuntawoot Jongrungklang ${ }^{2}$,
}

Pitakpong Maneerattanarungroj ${ }^{3} \&$ Narisa Kunpratum ${ }^{4}$

1. Salt Tolerance Rice Research Group, Department of Biology, Faculty of Science, Khon Kaen University, Khon Kaen, 40002, Thailand; worasitikulya@gmail.com, Thapakornr@kkumail.com

2. Northeast Thailand Cane and Sugar Research Center, Faculty of Agriculture, Khon Kaen University, Khon Kaen, 40002, Thailand; nuntawootjrk@gmail.com

3. Faculty of Veterinary Science, Khon Kaen University, Khon Kaen, 40002, Thailand; pitman@kku.ac.th

4. Department of Biology, Faculty of Science, Naresuan University, Pitsanulok, 65000, Thailand; lugmee@hotmail.com * Correspondence

\section{Received 11-III-2020. Corrected 06-VIII-2020. $\quad$ Accepted 18-VIII-2020.}

\begin{abstract}
Introduction: Drought is an important stress factor for sugarcane production in many areas of the world. Water proportion and moisture indices are applicable information for agronomic planning to forecast water excess or deficit during the crop cycle. Objective: Leaf anatomical features of two different sugarcane Saccharum 'UT12' (drought susceptible cultivar) and Saccharum 'UT13' (drought tolerant cultivar) were compared under early drought stress situation between 30 and 90 days after planting. Methods: Forty leaf anatomical features were investigated using peeling and free hand sectioning technique. Results: Some anatomical characteristics showed response to drought stress. Saccharum 'UT12' demonstrated higher sensitivity toward anatomical characteristics than Saccharum 'UT13'. A total of 23 and 15 out of the 40 anatomical characteristics showed significance in Saccharum 'UT12' and Saccharum 'UT13', respectively. Some anatomical features such as cell wall and cuticle thickness, vascular bundle size, stomatal size and density can be used as important markers for drought stress assessment in sugarcane leaf. Conclusions: This is the first report describing comparative leaf anatomy of sugarcane Saccharum 'UT12' and Saccharum 'UT13' in Thailand under drought stress. Results will provide important information to improve adaptation mechanisms of tolerant sugarcane cultivars under initial drought stress situations.
\end{abstract}

Key words: adaptation; drought stress; leaf anatomy; sugarcane; phenotypic correlation.

Taratima, W., Ritmaha, T., Jongrungklang, N., Maneerattanarungroj, P., \& Kunpratum, N. (2020). Effect of stress on the leaf anatomy of sugarcane cultivars with different drought tolerance (Saccharum officinarum, Poaceae). Revista de Biología Tropical, 68(4), 1159-1170.

Sugarcane (Saccharum spp.) is a globally important bioenergy crop (Vargas et al., 2014). Sugar, energy and surfactant are produced from sugarcane (Wiedenfeld, 2000). Many factors affect sugarcane yields including soil quality, cultivar, physiology, crop management and economic reasons (Santillán-Fernández et al., 2016). Drought is an important stress factor for sugarcane production in many areas (Santillán-Fernández et al., 2016). Climatic factors cannot be controlled but knowledge concerning historical regional meteorological conditions can be considered for optimal management of crop cycles (Van-Ittersum \& Rabbinge, 1997) and cultivar selection. Water proportion and moisture indices are applicable 
information for agronomic planning to forecast water excess or deficit during the crop cycle (Santillán-Fernández et al., 2016).

Most sugarcane in Thailand is grown under rain-fed environments (Laclau \& Laclau, 2009). Periods of drought regularly occur, especially during the early growing period. Sugarcane culture in Thailand occurs during October and November, or until the remaining moisture content in the soil is consumed. Drought affects plant growth and can decrease sugarcane yield by up to $60 \%$ (Robertson et al., 1999). Previous research reported the response of biochemical, physiological and morphological characteristics on drought tolerance criteria.

Under conditions of water deficit, plants show biochemical, physiological and molecular responses at both whole plant and cellular levels (Hasegawa et al., 2000). Osmotic stresses induced by drought environment also activate development and water situation disturbance causes ion transportation changes along with uptake schemes (Lutts, Kinet, \& Bouharmont, 1996; Bajji, Lutts, \& Kinet, 2000; Abbas, Ahmad, Sabir, \& Shah, 2014). Water absorption from the soil into plant roots uses osmosis mechanisms to balance pressure alteration. During drought stress conditions, plasma membranes are damaged by free radicals that induce electrolyte leakage. Drought stress also motivates plant adaptation for growth and survival (Boaretto et al., 2014). Plant cells adapt by reducing water potential, while carbon dioxide $\left(\mathrm{CO}_{2}\right)$ intensity within the palisade or spongy parenchyma decreases, causing low growth and photosynthetic rates (Shao, Chu, Jaleel, \&, Zhao, 2008). Scant information concerning the anatomical characteristics of sugarcane under early drought conditions is available in the literature. Under drought stress conditions, some anatomical features respond by reducing water content within the xylem, whereas pit of sclerenchymal cell walls are increases (Bosabalidis \& Kofdis, 2002). Bulliform cells enlarge and epidermal cells and leaf lamina become thicker. Stomatal density increases while size decreases (Nawazish, Hameed, \& Naurin, 2006; Taratima, Ritmaha, Jongrungklang,
Raso, \& Maneerattanarungroj, 2019). However, recent reports showed that bulliform cells of sugarcane Saccharum 'F127' and Saccharum 'YL6' did not expand under severe drought stress conditions (Zhang et al., 2015).

Morphological, physiological, biochemical and anatomical features of sugarcane families have been investigated to optimize crop yield. Previous studies proved that Saccharum 'UT13' which derived from wild-type genotype was assumed as the drought-tolerant cultivar (Palachai, Songsri, \& Jongrungklang, 2019; Khonghintaisong, Songsri, \& Jongrungklang, 2020), while Saccharum 'UT12' was identified susceptible to drought stress (Khonghintaisong, Songsri, \& Jongrungklang, 2020). Differences in their genetic background might provide a different anatomical characteristic after grown under drought stress condition. However, to the best of our knowledge, leaf anatomical features of sugarcane Saccharum 'UT12' and Saccharum 'UT13' have not been compared under initial drought stress situations. Information from this study can be used for sugarcane growth improvement and considerate to improve sugarcane genotypes in our breeding programs as parents selection for drought-resistance cultivars in the future.

\section{MATERIALS AND METHODS}

Plant materials and experimental details: Experiments were performed with potgrown plants between March and June 2018 at the Agronomy Field Crop Station, Faculty of Agriculture, Khon Kaen University, Khon Kaen, Thailand. Anatomical characteristics of the samples were investigated from July to December 2018. Two sugarcane cultivars, Saccharum 'UT12' and Saccharum 'UT13' with different drought tolerances were used (Palachai et al., 2019; Khonghintaisong et al., 2020).

Saccharum 'UT12' is a hybrid from commercial canes Saccharum 'Supanburi 80' $\mathrm{x}$ Saccharum 'UT3' (Mother x Father). Saccharum 'UT12' has been identified as having moderate tolerance to red rot wilt and smut diseases with moderate drought resistance. By contrast, 
Saccharum 'UT13' has been determined as sensitive to red rot wilt and smut diseases with good drought tolerance as a hybrid from $S$. spontaneum ' $\mathrm{BC}_{3}$ ' (wild type) $\mathrm{x}$ Saccharum 'UT8' (Mother x Father) (Office of the Cane and Sugar Board, 2015). Standard pots of size $80 \mathrm{~cm}$ height and $50 \mathrm{~cm}$ diameter were used in all treatments. $80 \mathrm{~kg}$ of dry soil were filled as four layers, $20 \mathrm{~kg}$ per layer and compared to achieve density at $1.55 \mathrm{~g} / \mathrm{cm}^{3}$. Soil properties were $77.9 \%$ sand, $20.0 \%$ silt, $2.07 \%$ clay, $11.3 \%$ Field capacity (FC), $4.06 \%$ Permanent wilting point (PWP), $0.337 \%$ Organic Matter (OM), pH 6.08 and 0.26 ds/m EC (Khonghintaisong, 2018).

A one bud sugarcane culm was used as the explant in all treatments, one culm per pot. Basal fertilizers at $50 \mathrm{~kg} \mathrm{~N}, 50 \mathrm{~kg} \mathrm{P}$ and 25 $\mathrm{kg} \mathrm{K} \mathrm{ha}{ }^{-1}$ were applied during growth in field conditions. Insects and weeds were controlled during the experimental period.

A full water scheme was applied for seedling growth in all treatment for 30 days after planting culture. For the control (non-water stress treatment), soil moisture was applied continuously and recorded throughout the experimental period, while for drought treatment, water was restricted from 30-90 days after planting cultures. Total water applied in the experiment followed crop water requirements calculated by Jangpromma et al. (2010) as follows:

$$
\text { ETcrop }=\text { ETo } \mathrm{K}_{\mathrm{c}}
$$

where ETcrop represents crop water requirement ( $\mathrm{mm} /$ day), ETo represents evapotranspiration of a reference crop under specified conditions calculated by the evaporation pan method, and $\mathrm{K}_{\mathrm{c}}$ is the crop water requirement coefficient for sugarcane.

Leaf anatomy studies: The third or fourth mature leaves from the shoots of control plants and treatments were collected at 90 days after culture. Approximately $160 \mathrm{~cm}$ long leaves were selected. The middle part of leaf blade $(10 \mathrm{~cm})$ was cut out and fixed in $100 \mathrm{ml}$ of
FAA70 fixative (70 \% ethyl alcohol, acetic acid, formaldehyde; 90:5:5) for anatomical studies. Three leaf areas as leaf margin, midrib and lamina were collected and dissected into small pieces before soaking in $15 \%(\mathrm{v} / \mathrm{v})$ Clorox (sodium hypochlorite) for 24 hours before peeling. Adaxial and abaxial epidermis were stained by $1 \%(w / v)$ Safranin O in ethyl alcohol before dehydration by serial ethyl alcohol and xylene and mounted using DePeX (Taratima et al., 2019).

For leaf peeling, both short and long epidermal cells, stomatal density, stomatal size (guard cell and subsidiary cell) were measured under a light compound microscope. Three areas of the leaf blade were free hand transverse sectioned before Safranin O staining, then dehydrated using serial ethyl alcohol and xylene and mounted using DePeX. Anatomical features were studied and recorded using a light compound microscope (Olympus $\mathrm{CH} 30$ ) and a Zeiss 5402140000004 using the MB2004 configuration AxioVision program. Forty anatomical characteristics were scored based on Nawazish et al. (2006), Zhang et al. (2015) and Taratima et al. (2019) as presented in Table 1 and Table 2.

Data analysis: At least five replications were examined in each treatment. The paired sample t-test was used for statistical significance (Taratima et al., 2019). Relationships between anatomical features were investigated using simple correlation. Anatomical traits were determined using the drought tolerance index (DTI) to compare values under drought stress conditions, with values for the field capacity condition based on Nautiyal, Nageswara Rao, and Joshi (2002) (more than $1=$ increase, less than $1=$ decrease) as follows:

\section{DTI $=$ Data of stress treatment/ \\ Data of non-stress treatment}

Correlation studies: Anatomical trait associations were calculated using correlation coefficients among interesting pairs of anatomical characteristics at phenotypic levels. 
Phenotypic correlation coefficients were calculated according to Searle (1961) and Singh et al. (2018) as follows:

Phenotypic correlation coefficients $(\mathrm{rp})=\frac{\operatorname{cov} \cdot \mathrm{XP}(\mathrm{p})}{\sqrt{\text { var. Xp. var. Yp }}}$

where Cov.XY (p) represents phenotypic covariance between characteristics $\mathrm{X}$ and $\mathrm{Y}$ and Var.X (p), and Var.Y (p) represent variance for characteristics $\mathrm{X}$ and $\mathrm{Y}$ at phenotypic levels. Data analysis was carried out using the SPSS version 19.

\section{RESULTS}

Transverse sections of unstressed and stressed sugarcane leaf Saccharum 'UT12' and Saccharum 'UT13' showed Kranz anatomy characteristics of $\mathrm{C} 4$ plants, with chlorenchymatous bundle sheaths and radially arranged mesophyll cells (Fig. 1A, 1B, 1C, 1D). Vascular bundles exhibited angular in outline or basic bundle type (Fig. 1E, 1F, 1G, 1H). Forty anatomical characteristics of Saccharum 'UT12' and Saccharum 'UT13' were measured as shown in Table 1 and Table 2, respectively. Lamina thickness of Saccharum 'UT12'

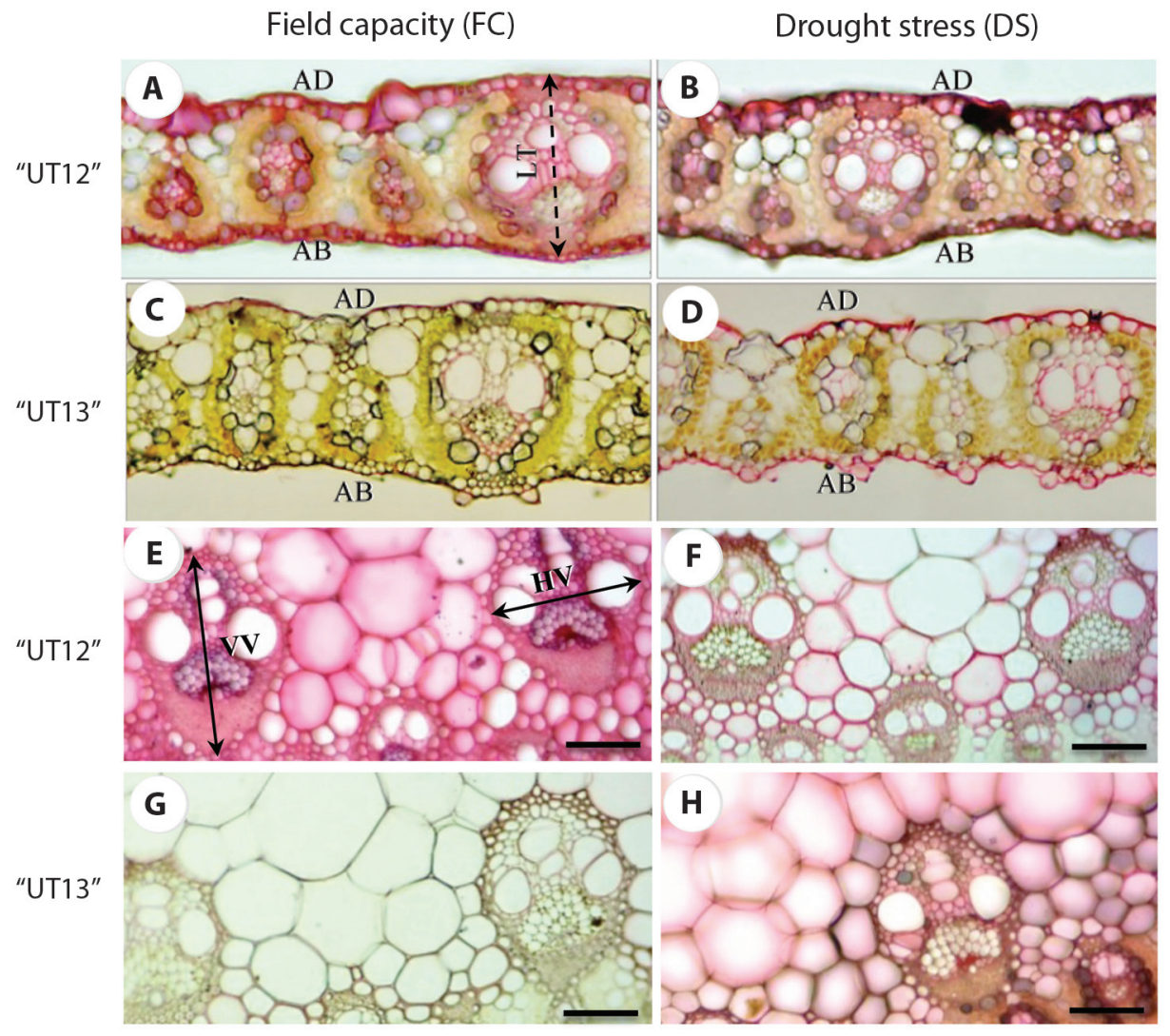

Fig. 1. Transverse section of sugarcane leaf comparing thickness and vascular bundle size from sugarcane with field capacity (FC) (non-water stress treatment) and drought stress (DS). A. leaf thickness of Saccharum 'UT12' with FC; B. leaf thickness of Saccharum 'UT12' with DS; C. leaf thickness of Saccharum 'UT13' with FC; D. leaf thickness of Saccharum 'UT13' with DS; E. vascular bundle size of Saccharum 'UT12' with FC; F. vascular bundle size of Saccharum 'UT12' with DS; G. vascular bundle size of Saccharum 'UT13' with FC; H. vascular bundle size of Saccharum 'UT13' with DS. (LT-leaf thickness; $A D$-adaxial; $A B$-abaxial; $V V$-vertical length of major vascular bundle; $H P$-horizontal length of major vascular bundle). Scale $=100 \mu \mathrm{m}$. 
and Saccharum 'UT13' decreased when grown under drought condition, while cell wall and cuticle thickness of lamina epidermal cells of Saccharum 'UT12' increased on both adaxial and abaxial sides, except in Saccharum 'UT13'. Almost all major vascular bundle features in the midrib of Saccharum 'UT12', and some characteristics in Saccharum 'UT13' enlarged after treatment $(\mathrm{P}<0.05)$. Major vascular bundle size in lamina blades of Saccharum 'UT12' and Saccharum 'UT13' showed differences after treatment; both vertical and horizontal length of Saccharum 'UT12' decreased while in Saccharum 'UT13' these significantly increased (Table 1 and Table 2).

Epidermal features of sugarcane leaf Saccharum 'UT12' and Saccharum 'UT13' were similar to grasses with mainly in small groups of specially large cells of bulliform cells (Fig. 2A, 2B, 2C, 2D) and regular patterns of short cells and long cells (Fig. 2E, 2F, 2G, 2H). Plant tissue type and alignment patterns were similar between unstressed and stressed leaves. Anatomical characteristics of sugarcane concurred with Metcalfe (1960) and Joarder, Roy, Sima, and Parvin (2010). Short cells of abaxial epidermis were shown between the midrib; almost all were solitary with some paired. Crossshaped silica bodies intermediated among cross or dumb-bell shapes (Fig. 2E, 2F, 2G, 2H). Stomata occurred with two subsidiary cells of triangular shape. All stomata appeared in the stomatal zone. Long-cells occurred closely alongside and above the midrib. Inter-stomatal cells showed indented ends including narrow and profound concavities.

Stomatal density of Saccharum 'UT12' was not significantly different in unstressed and stressed condition, while Saccharum 'UT13' showed significant differences in stomatal density of unstressed and stressed condition on both adaxial and abaxial sides. Stomatal size of almost all stressed leaves of Saccharum 'UT12' increased, while Saccharum 'UT13' showed decrease in both adaxial and abaxial sides. Stomatal size and density were related to drought adaptation.
Nine anatomical features were calculated for phenotypic correlation coefficient estimation (Table 3). Lamina thickness of Saccharum 'UT12 and Saccharum 'UT13' showed positive correlation with MCC-AD (Midrib cell wall and cuticle thickness-Ad), LCC-AD (Lamina cell wall and cuticle thickness -Ad) and BULH (Bulliform cell horizontal length).

\section{DISCUSSION}

Decreasing leaf thickness was an important marker for drought tolerance in sugarcane leaf concurring with our previous study on Saccharum 'KK3' (Taratima et al., 2019). Zhang et al. (2015) revealed that some anatomical features such as lower or abaxial cuticle thickness of leaf epidermis were appropriate to examine drought stress level of Saccharum 'F127' and Saccharum 'YL6'. Saccharum 'UT12' and 'UT13' showed some drought-resistant characters same as in Saccharum 'F127' (strongly drought-resistant cultivar) in term of lower epidermis cuticle thickness was getting thickened under drought stress condition. Moreover, changing of this anatomical character was similar to the studied of Saccharum 'KK3' which is a drought tolerance cultivar that abaxial cuticle thickness of leaf epidermis was clearly increased during water stress situation (Taratima et al., 2019). However, diverse species or cultivars should be assessed for anatomical adaptation features (Graca et al., 2010). Generally, leaf thickness was associated with midrib and lamina vascular bundle increase. In this study, almost all anatomical characteristics of major vascular bundles of midrib and lamina were significantly different in Saccharum 'UT12', while only several characteristics were significant in Saccharum 'UT13'. All major vascular bundle features of lamina in Saccharum 'UT12' decreased under drought condition, appropriate for reduced water transportation due to leaf area reduction. After reduction of water content in plant cells, turgor pressure and cell volume decreased, weakening cell walls. If this phenomenon occurs continually for a long time, cells compact and higher 


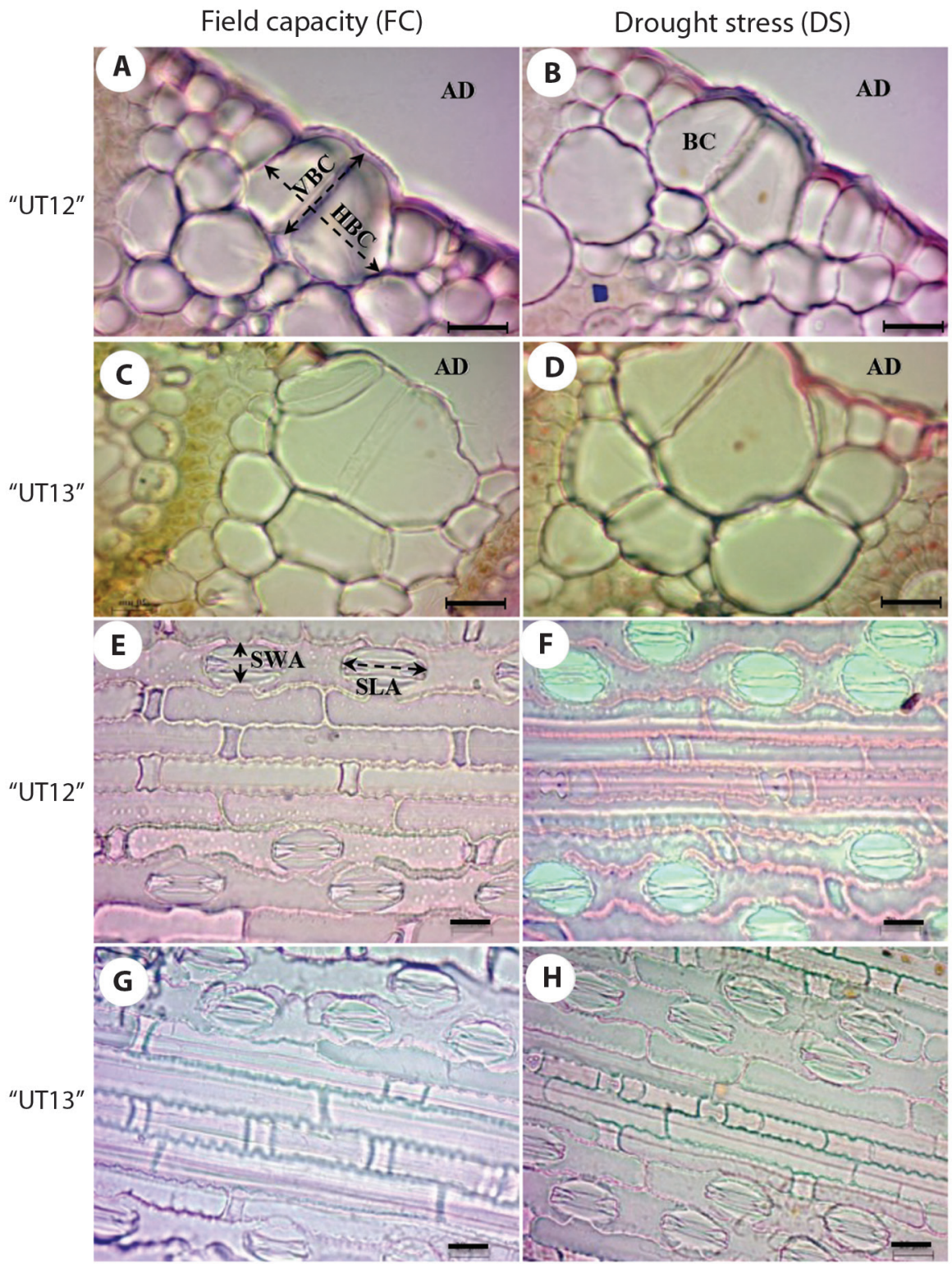

Fig. 2. Transverse section of sugarcane leaf displaying bulliform cell size of field capacity (FC) and drought stress (DS) from 30-90 days after first ratoon A. cell wall of epidermal cell and cuticle thickness of Saccharum 'UT12' with FC; B. Saccharum 'UT12' with DS; C. cell wall of epidermal cell and cuticle thickness of Saccharum 'UT13' with FC; D. Saccharum 'UT13' with DS; E. stomata and epidermal cell of Saccharum 'UT12' with FC; F. Saccharum 'UT12' with DS; G. stomata and epidermal cell of Saccharum 'UT13' with FC; H. stomata and epidermal cell of Saccharum 'UT13' with DS ( $A D$-adaxial; $B C$-bulliform cell; $V B C$-vertical length of bulliform cell; $H B C$-horizontal length of bulliform cell; $S W A$ stomatal width of adaxial side; $S L A$-stomatal length of adaxial side). Scale $=20 \mu \mathrm{m}$. 
TABLE 1

Assessment of anatomical features of sugarcane leaf Saccharum 'UT12' under unstressed and early stress situations

\begin{tabular}{|c|c|c|c|c|}
\hline Character size $(\mu \mathrm{m})$ & Unstressed $(\bar{x} \pm \mathrm{SD})$ & Stressed $(\bar{x} \pm S D)$ & DTI & Sig. \\
\hline Lamina thickness & $195.3 \pm 18.3$ & $169.0 \pm 12.2$ & 0.86 & $*$ \\
\hline \multicolumn{5}{|l|}{ Midrib-epidermal cell } \\
\hline Cell wall \& cuticle thickness (ad) & $3.6 \pm 0.7$ & $3.4 \pm 1.7$ & 0.94 & - \\
\hline Cell wall \& cuticle thickness (ab) & $3.4 \pm 1.0$ & $3.5 \pm 0.7$ & 1.02 & - \\
\hline \multicolumn{5}{|l|}{ Lamina-epidermal cell } \\
\hline Cell wall \& cuticle thickness (ad) & $3.9 \pm 0.9$ & $5.3 \pm 0.4$ & 1.35 & $*$ \\
\hline Cell wall \& cuticle thickness (ab) & $1.7 \pm 0.5$ & $3.8 \pm 0.6$ & 2.23 & $* *$ \\
\hline \multicolumn{5}{|l|}{ Major vascular bundle of midrib } \\
\hline Vertical length & $128.9 \pm 5.0$ & $167.3 \pm 8.8$ & 1.29 & $* *$ \\
\hline Horizontal length & $186.2 \pm 4.1$ & $191.9 \pm 9.5$ & 1.03 & - \\
\hline $1^{\text {st }}$ vessel diameter (metaxylem) & $59.2 \pm 8.9$ & $60.3 \pm 2.7$ & 1.01 & - \\
\hline $2^{\text {nd }}$ vessel diameter (metaxylem) & $62.9 \pm 6.2$ & $62.1 \pm 3.7$ & 0.98 & - \\
\hline Vessel cell wall thickness (protoxylem) & $4.3 \pm 0.6$ & $6.5 \pm 1.6$ & 1.51 & * \\
\hline Phloem vertical length & $56.3 \pm 7.5$ & $61.3 \pm 7.3$ & 1.08 & - \\
\hline Phloem horizontal length & $95.1 \pm 9.7$ & $120.3 \pm 8.2$ & 1.26 & * \\
\hline Bundle sheath extension length & $132.2 \pm 8.1$ & $120.7 \pm 5.8$ & 0.91 & $*$ \\
\hline \multicolumn{5}{|l|}{ Major vascular bundle of lamina } \\
\hline Vertical length & $115.1 \pm 11.1$ & $89.0 \pm 11.4$ & 0.77 & $* *$ \\
\hline Horizontal length & $128.0 \pm 28.1$ & $106.5 \pm 20.3$ & 0.83 & - \\
\hline $1^{\text {st }}$ vessel diameter (metaxylem) & $48.1 \pm 8.2$ & $37.6 \pm 5.9$ & 0.78 & * \\
\hline $2^{\text {nd }}$ vessel diameter (metaxylem) & $47.6 \pm 1.6$ & $34.2 \pm 7.2$ & 0.71 & * \\
\hline Vessel cell wall thickness (protoxylem) & $3.7 \pm 0.8$ & $3.6 \pm 0.6$ & 0.97 & - \\
\hline Phloem vertical length & $41.4 \pm 1.4$ & $32.4 \pm 5.1$ & 0.78 & * \\
\hline Phloem horizontal length & $63.2 \pm 3.5$ & $50.5 \pm 9.8$ & 0.79 & $*$ \\
\hline \multicolumn{5}{|l|}{ Other characters } \\
\hline Bulliform cell vertical length & $56.9 \pm 3.6$ & $38.9 \pm 6.9$ & 0.68 & $*$ \\
\hline Bulliform cell horizontal length & $81.5 \pm 26.0$ & $58.1 \pm 8.4$ & 0.71 & * \\
\hline Stomatal width (ad) & $22.0 \pm 1.7$ & $23.3 \pm 1.2$ & 1.05 & - \\
\hline Stomatal length (ad) & $36.3 \pm 10.4$ & $34.0 \pm 1.4$ & 0.93 & - \\
\hline Stomatal width $(\mathrm{ab})$ & $20.4 \pm 0.7$ & $24.4 \pm 1.5$ & 1.19 & ** \\
\hline Stomatal length (ab) & $28.0 \pm 9.0$ & $35.7 \pm 1.7$ & 1.27 & $* *$ \\
\hline Interstomatal cell width (ad) & $19.5 \pm 1.5$ & $17.6 \pm 2.1$ & 0.90 & $*$ \\
\hline Interstomatal cell length (ad) & $21.8 \pm 7.5$ & $42.2 \pm 15.2$ & 1.93 & $*$ \\
\hline Interstomatal cell width (ab) & $22.5 \pm 2.8$ & $22.1 \pm 2.4$ & 0.98 & - \\
\hline Interstomatal cell length (ab) & $15.8 \pm 2.9$ & $25.5 \pm 5.8$ & 1.61 & $* *$ \\
\hline Short-cell width (ad) & $15.8 \pm 2.4$ & $19.3 \pm 1.4$ & 1.22 & $*$ \\
\hline Short-cell length (ad) & $7.1 \pm 1.2$ & $8.8 \pm 1.5$ & 1.23 & $*$ \\
\hline Short-cell width (ab) & $11.9 \pm 1.6$ & $16.8 \pm 2.7$ & 1.41 & $* *$ \\
\hline Short-cell length $(a b)$ & $9.0 \pm 2.5$ & $8.4 \pm 1.9$ & 0.93 & - \\
\hline Long-cell width (ad) & $14.7 \pm 0.9$ & $16.6 \pm 1.5$ & 1.12 & - \\
\hline Long-cell length (ad) & $109.0 \pm 19.3$ & $108.2 \pm 11.2$ & 0.99 & - \\
\hline Long-cell width (ab) & $12.2 \pm 1.8$ & $15.5 \pm 1.1$ & 1.27 & ** \\
\hline Long-cell length (ab) & $96.7 \pm 8.3$ & $102.8 \pm 20.8$ & 1.06 & - \\
\hline Stomatal density (ad) $\left(\mathrm{No} / \mathrm{mm}^{2}\right)$ & $195.6 \pm 24.0$ & $195.6 \pm 55.2$ & 1.00 & - \\
\hline Stomatal density $(\mathrm{ab})\left(\mathrm{No} / \mathrm{mm}^{2}\right)$ & $219.0 \pm 48.2$ & $230.7 \pm 79.6$ & 1.05 & - \\
\hline
\end{tabular}

* Significant difference at $\mathrm{P}<0.05, * *$ Significant difference at $\mathrm{P}<0.001 ; a b$-abaxial; $a d$-adaxial. (DTI $=$ drought tolerance index). 
TABLE 2

Assessment of anatomical features of sugarcane leaf Saccharum 'UT13' under unstressed and early stress situations

\begin{tabular}{|c|c|c|c|c|}
\hline Character size $(\mu \mathrm{m})$ & Unstressed $(\bar{x} \pm \mathrm{SD})$ & Stressed $(\bar{x} \pm \mathrm{SD})$ & DTI & Sig. \\
\hline Lamina thickness & $198.8 \pm 38.4$ & $191.5 \pm 11.8$ & 0.96 & - \\
\hline \multicolumn{5}{|l|}{ Midrib-epidermal cell } \\
\hline Cell wall \& cuticle thickness (ad) & $2.2 \pm 0.4$ & $2.3 \pm 0.4$ & 1.04 & - \\
\hline Cell wall \& cuticle thickness (ab) & $2.7 \pm 0.4$ & $3.3 \pm 0.4$ & 1.22 & $*$ \\
\hline \multicolumn{5}{|l|}{ Lamina-epidermal cell } \\
\hline Cell wall \& cuticle thickness (ad) & $5.0 \pm 0.8$ & $4.2 \pm 0.9$ & 0.84 & - \\
\hline Cell wall \& cuticle thickness (ab) & $3.8 \pm 1.1$ & $3.2 \pm 0.7$ & 0.84 & - \\
\hline \multicolumn{5}{|l|}{ Major vascular bundle of midrib } \\
\hline Vertical length & $138.8 \pm 11.4$ & $152.9 \pm 5.9$ & 1.10 & $*$ \\
\hline Horizontal length & $158.2 \pm 7.8$ & $168.3 \pm 10.6$ & 1.06 & * \\
\hline $1^{\text {st }}$ vessel diameter (metaxylem) & $50.5 \pm 3.0$ & $52.8 \pm 4.1$ & 1.04 & - \\
\hline $2^{\text {nd }}$ vessel diameter (metaxylem) & $51.1 \pm 3.3$ & $52.0 \pm 2.4$ & 1.01 & - \\
\hline Vessel cell wall thickness (protoxylem) & $4.3 \pm 0.8$ & $4.2 \pm 0.5$ & 0.97 & - \\
\hline Phloem vertical length & $51.6 \pm 6.8$ & $54.8 \pm 3.1$ & 1.06 & - \\
\hline Phloem horizontal length & $107.8 \pm 8.4$ & $117.9 \pm 7.3$ & 1.09 & - \\
\hline Bundle sheath extension length & $105.8 \pm 9.0$ & $123.0 \pm 11.4$ & 1.16 & $*$ \\
\hline \multicolumn{5}{|l|}{ Major vascular bundle of lamina } \\
\hline Vertical length & $94.9 \pm 13.1$ & $104.4 \pm 14.2$ & 1.10 & \\
\hline Horizontal length & $119.0 \pm 5.2$ & $112.0 \pm 12.2$ & 0.94 & \\
\hline First metaxylem diameter & $40.9 \pm 3.7$ & $36.3 \pm 5.0$ & 0.88 & - \\
\hline Second metaxylem diameter & $41.5 \pm 4.0$ & $39.2 \pm 5.0$ & 0.93 & - \\
\hline Protoxylem cell wall thickness & $2.8 \pm 0.7$ & $3.3 \pm 0.7$ & 1.17 & - \\
\hline Phloem vertical length & $40.4 \pm 3.8$ & $33.3 \pm 7.9$ & 0.82 & $*$ \\
\hline Phloem horizontal length & $70.6 \pm 4.7$ & $53.0 \pm 5.4$ & 0.75 & $*$ \\
\hline \multicolumn{5}{|l|}{ Other characters } \\
\hline Bulliform cell vertical length & $50.0 \pm 13.3$ & $61.7 \pm 12.9$ & 1.23 & - \\
\hline Bulliform cell horizontal length & $64.7 \pm 5.1$ & $83.1 \pm 13.7$ & 1.28 & $*$ \\
\hline Stomatal width (ad) & $24.8 \pm 2.2$ & $21.0 \pm 1.3$ & 0.84 & $* *$ \\
\hline Stomatal length (ad) & $37.4 \pm 2.3$ & $32.3 \pm 5.7$ & 0.86 & $*$ \\
\hline Stomatal width (ab) & $25.9 \pm 1.5$ & $23.4 \pm 3.8$ & 0.90 & - \\
\hline Stomatal length $(\mathrm{ab})$ & $32.9 \pm 3.7$ & $35.0 \pm 1.0$ & 1.06 & - \\
\hline Interstomatal cell width (ad) & $22.2 \pm 2.6$ & $19.2 \pm 1.3$ & 0.86 & $*$ \\
\hline Interstomatal cell length (ad) & $31.1 \pm 7.3$ & $25.5 \pm 3.8$ & 0.81 & - \\
\hline Interstomatal cell width (ab) & $19.9 \pm 3.0$ & $21.4 \pm 3.4$ & 1.07 & - \\
\hline Interstomatal cell length $(\mathrm{ab})$ & $28.1 \pm 11.1$ & $25.7 \pm 7.3$ & 0.91 & - \\
\hline Short-cell width (ad) & $14.8 \pm 3.3$ & $11.4 \pm 2.8$ & 0.77 & - \\
\hline Short-cell length (ad) & $7.8 \pm 2.0$ & $8.9 \pm 2.2$ & 1.14 & - \\
\hline Short-cell width (ab) & $14.6 \pm 3.0$ & $11.8 \pm 3.4$ & 0.80 & $*$ \\
\hline Short-cell length (ab) & $9.4 \pm 3.2$ & $6.2 \pm 0.9$ & 0.65 & $* *$ \\
\hline Long-cell width (ad) & $14.1 \pm 1.79$ & $9.0 \pm 2.3$ & 0.63 & - \\
\hline Long-cell length (ad) & $119.7 \pm 34.5$ & $112.5 \pm 16.2$ & 0.93 & - \\
\hline Long-cell width (ab) & $16.3 \pm 2.4$ & $11.0 \pm 2.1$ & 0.67 & $* *$ \\
\hline Long-cell length (ab) & $112.2 \pm 19.4$ & $101.3 \pm 10.9$ & 0.90 & - \\
\hline Stomatal density (ad) (No/mm²) & $131.4 \pm 37.7$ & $315.4 \pm 61.2$ & 2.40 & $* *$ \\
\hline Stomatal density $(\mathrm{ab})\left(\mathrm{No} / \mathrm{mm}^{2}\right)$ & $157.7 \pm 51.8$ & $332.9 \pm 34.2$ & 2.11 & $* *$ \\
\hline
\end{tabular}

* Significant difference at $\mathrm{P}<0.05 * *$ Significant difference at $\mathrm{P}<0.001 ; a b$-abaxial; $a d$-adaxial. (DTI $=$ drought tolerance index). 
TABLE 3

Estimates of phenotypic correlation coefficients between 9 traits in sugarcane Saccharum 'UT12' and Saccharum 'UT13' under drought situation

\begin{tabular}{|c|c|c|c|c|c|c|c|c|}
\hline Character & LT & MCC-AD & LCC-AD & MVB-V & MVB-H & VCWT & BDS-EXL & BULH \\
\hline & \multicolumn{8}{|c|}{ UT12 } \\
\hline STD-AB & -0.053 & -0.541 & 0.110 & -0.475 & -0.364 & $-0.648^{*}$ & -0.314 & 0.387 \\
\hline LT & & $0.661^{*}$ & 0.057 & -0.229 & -0.120 & -0.164 & 0.506 & 0.033 \\
\hline MCC-AD & & & -0.155 & -0.143 & 0.003 & 0.432 & $0.703^{*}$ & 0.086 \\
\hline LCC-AD & & & & 0.107 & -0.306 & -0.345 & 0.191 & 0.319 \\
\hline MVB-V & & & & & $0.823 * *$ & 0.110 & -0.024 & 0.223 \\
\hline MVB-H & & & & & & 0.161 & -0.099 & -0.226 \\
\hline VCWT & & & & & & & 0.074 & -0.190 \\
\hline \multirow[t]{2}{*}{ BDS-EXL } & & & & & & & & 0.609 \\
\hline & \multicolumn{8}{|c|}{ UT13 } \\
\hline STD-AB & 0.126 & 0.504 & $0.745^{*}$ & -0.607 & $-0.636^{*}$ & -0.343 & -0.073 & 0.124 \\
\hline LT & & 0.202 & 0.029 & -0.131 & -0.275 & -0.233 & -0.071 & 0.561 \\
\hline MCC-AD & & & 0.175 & -0.529 & $-0.735 * *$ & -0.106 & -0.277 & -0.222 \\
\hline LCC-AD & & & & -0.431 & -0.165 & -0.261 & -0.379 & -0.075 \\
\hline MVB-V & & & & & 0.235 & 0.302 & 0.197 & -0.122 \\
\hline MVB-H & & & & & & 0.315 & -0.147 & -0.056 \\
\hline VCWT & & & & & & & -0.611 & $-0.653 *$ \\
\hline BDS-EXL & & & & & & & & $0.671 *$ \\
\hline
\end{tabular}

* Significant correlation at $\mathrm{P}<0.05$, ** Significant correlation at $\mathrm{P}<0.001$.

(LT- Lamina thickness; MCC-AD- Midrib cell wall and cuticle thickness (Ad); LCC-AD- Lamina cell wall and cuticle thickness (Ad); MVB-V- Midrib major vascular bundle (vertical length); MVB-H- Midrib major vascular bundle (horizontal length); VCWT-Vessel cell wall thickness; BDS-EXL- Bundle sheath extension length; BULH-Bulliform cell horizontal length; STD-AB- Stomatal density (ab).

solute concentrations cause drought stress sensitivity of plant growth (Udomprasert, 2015).

Transportation of water and food via tracheal elements was related to the photosynthetic rate. Lack of water during plant growth inhibited transportation of water and food by turgor pressure reduction (Taiz \& Zeiger, 2002). Bundle-sheath cells and vascular bundle sizes were associated with respiration and photosynthetic percentage (Wu, Liu, Wang, Zhou, \& Chen, 2011). Vascular bundle size enlargement improved water and food transportation efficiency (Bosabalidis \& Kofdis, 2002).

Plant adaptation with heed to water retention was revealed by leaf area reduction. Epidermal and mesophyll cells decreased in size, whereas cell density also increased due to reduction of transpiration and respiration rate (Bosabalidis \& Kofdis, 2002). Under drought stress situations, cell expansion disruption resulted in deceleration of leaf extension, causing interruption of $\mathrm{H}$-ion movement across cell membranes. Cell and leaf expansion postponement also affected transpiration rate reduction and helped to preserve water content within the cells during drought stress conditions (Udomprasert, 2015). Some studies revealed that leaf thickness was associated with photosynthetic rate and plant growth under drought stress conditions. Generally, leaf thickness increased in drought tolerant plants under drought stress environments, resulting in increased mesophyll density (Ngernmuen, 2013; Kulya et al., 2014).

Closing of stomata in drought stress environments caused transpiration rate reduction that reduced photosynthetic rates and $\mathrm{CO}_{2}$ fixation (Taiz \& Zeiger, 2002). Nevertheless, more factors affected the photosynthetic rate. Some reports revealed no significant difference in photosynthetic rates for plant growth 
under drought stress conditions and showed anatomical traits of leaf thickness decreasing, stomatal density increasing or stomatal size decreasing (Bosabalidis \& Kofdis, 2002; Nawazish et al., 2006).

Bulliform cell size of Saccharum 'UT12' significantly decreased after treatment but increased in Saccharum 'UT13', especially in horizontal length $(\mathrm{P}<0.05)$. This finding concurred with Nawazish et al. (2006) who determined that bulliform plant cells expanded as an anatomical adaptation to drought stress of Cenchrus ciliaris L. leaf.

Responsiveness of stomata to drought condition varied in some plant species or cultivars (Graca et al., 2010). Drought stress tolerant plants also exhibited high photosynthetic rates. Photosystem II functioning still constant as long as experimental periods causes of protein protection ( $\mathrm{Lu} \&$ Zhang, 1999). Hence, the photosynthetic process was advocated by stomatal density increasing and stomatal size decreasing (Nawazish et al., 2006). This suggested that Saccharum 'UT13' had higher drought tolerance than Saccharum 'UT12'.

Nawazish et al. (2006) and Taratima et al. (2019) reported that increasing bulliform cells and lamina thickness were related to anatomical adaptation under drought. When the correlation between lamina thickness and bulliform cell size was compared, Saccharum 'UT13' showed higher positive correlation than Saccharum 'UT12'. Abaxial stomatal density presented negative correlation with lamina thickness in Saccharum 'UT12', while positive correlation in Saccharum 'UT13'. This positive correlation indicated that increasing lamina thickness may expand bulliform cells. This result agreed with Bosabalidis and Kofdis, (2002), Nawazish et al. (2006), Ngernmuen, (2013) and Kulya et al. (2014) who reported on plant adaptation based on anatomical features. Although anatomical characteristics in drought stress conditions were investigated in some previous reports, their anatomical correlations remain poorly understood. However, the correlation of some anatomical characteristics may not be persistent due to several factors such as diverse cultivar, developmental stage, climate change or environmental management.

Ethical statement: authors declare that they all agree with this publication and made significant contributions; that there is no conflict of interest of any kind; and that we followed all pertinent ethical and legal procedures and requirements. All financial sources are fully and clearly stated in the acknowledgements section. A signed document has been filed in the journal archives.

\section{ACKNOWLEDGMENTS}

This study was funded by the Northeast Thailand Cane and Sugar Research Center, Faculty of Agriculture, Khon Kaen University, Thailand. We acknowledge the Department of Biology, Faculty of Science, Khon Kaen University for facility support.

\section{RESUMEN}

Efecto del estrés sobre la anatomía foliar de cultivares de caña de azúcar con diferente tolerancia a la sequía (Saccharum officinarum, Poaceae). Introducción: La sequía es un factor de estrés importante para la producción de caña de azúcar en muchas áreas del mundo. La proporción de agua y los índices de humedad son información aplicable en la planificación agronómica para pronosticar el exceso o el déficit de agua durante el ciclo del cultivo. Objetivo: Se compararon las características anatómicas de las hojas de dos cañas de azúcar diferentes Saccharum 'UT12' (cultivar susceptible a la sequía) y Saccharum 'UT13' (cultivar tolerante a la sequía) bajo una situación de estrés por sequía temprana entre 30 y 90 días después de la siembra. Métodos: Se investigaron las características anatómicas de cuarenta hojas utilizando la técnica de seccionamiento de pelado y manos libres. Resultados: Algunas características anatómicas mostraron respuesta a estrés por sequía. Saccharum "UT12" demostró una mayor sensibilidad hacia las características anatómicas que Saccharum 'UT13'. Un total de 23 y 15 de las 40 características anatómicas mostraron significancia en Saccharum 'UT12' y Saccharum 'UT13', respectivamente. Algunas características anatómicas como la pared celular y el grosor de la cutícula, el tamaño del haz vascular, el tamaño y la densidad de los estomas se pueden utilizar como marcadores importantes para evaluar el estrés por sequía en la hoja de caña de azúcar. Conclusiones: Este es el primer reporte que describe la anatomía comparada de la hoja de la caña 
de azúcar Saccharum 'UT12' y Saccharum 'UT13' en Tailandia bajo estrés por sequía. Los resultados proporcionarán información importante para mejorar los mecanismos de adaptación de cultivares tolerantes de caña de azúcar bajo situaciones iniciales de estrés por sequía.

Palabras clave: adaptación; estrés por sequía; anatomía de la hoja; caña de azúcar; correlación fenotípica.

\section{REFERENCES}

Abbas, R.S., Ahmad, D.S., Sabir, M.S., \& Shah, H.A. (2014). Detection of drought tolerant sugarcane genotypes (Saccharum officinarum) using lipid peroxidation, antioxidant activity, glycine-betaine and proline contents. Journal of Soil Science and Plant Nutrition, 14, 233-243.

Bajji, M., Lutts, S., \& Kinet, J.M. (2000). Physiological changes after exposure to and recovery from polyethylene glycol-induced water deficit in callus cultures issued from durum wheat (Triticum durum Desf.) cultivars differing in drought resistance. Journal of Plant Physiology, 156, 75-83.

Boaretto, L.F., Carvalho, G., Borgo, L., Creste, S., Landell, M.G.A., Mazzafera, P., \& Azevedo, R.A. (2014). Water stress reveals differential antioxidant responses of tolerant and non-tolerant sugar cane genotypes. Plant Physiology and Biochemistry, 74, 165-175.

Bosabalidis, A.M., \& Kofidis, A. (2002). Comparative effects of drought stress on leaf anatomy of two olive cultivars. Plant Science, 163, 375-379.

Graca, J.P.D., Rodrigues, F.A., Farias, J.R.B., Oliveira, M.C.N.D., Hoffmann-Campo, C.B., \& Zingaretti, S.M. (2010). Physiological parameters in sugar cane cultivars submitted to water deficit. Brazilian Journal of Plant Physiology, 22, 189-197.

Hasegawa, P., Bressan, R.A., Zhu, J.K., \& Bohnert, H.J. (2000). Plant cellular and molecular to high salinity. Annual Review of Plant Physiology and Plant Molecular Biology, 51, 463-499.

Jangpromma, N., Kitthaisong, S., Lomthaisong, K., Daduang, S., Jaisil, P., \& Thammasirirak, S. (2010). A Proteomics Analysis of Drought Stress-Responsive Proteins as Biomarker for Drought-Tolerant Sugar cane Cultivars. American Journal of Biochemistry and Biotechnology, 6, 89-102.

Joarder, N., Roy, K.A., Sima, N.S., \& Parvin, K. (2010). Leaf blade and midrib anatomy of two sugarcane cultivars of Bangladesh. Journal of Biosciences, $18,66-73$.

Khonghintaisong, J. (2018). Physiological characteristics involved with tiller development to millable cane and responses of rooting and physiological traits to early season drought conditions in sugarcane
(Master's Thesis). Khon Kaen University, Khon Kaen, Thailand.

Khonghintaisong, J., Songsri, P., \& Jongrungklang, N. (2020). Root characteristics of individual tillers and the relationships with above-ground growth and dry matter accumulation in sugarcane. Pakistan Journal of Botany, 52, 101-109.

Kulya, C., Theerakulpisut, P., Sriyot, N., Pattanagul, W., Lontom, W., Sanitchon, J., Pengrat, J., Siangliw, L.J., \& Toojinda, T. (2014). Comparative leaf anatomy of drought-tolerant and drought-sensitive rice cultivars. Thai Journal of Botany, 6, 95-105.

Laclau, P.B., \& Laclau, J.P. (2009). Growth of the whole root system for a plant crop of sugarcane under rainfed and irrigated environments in Brazil. Field Crops Research, 114, 351-360.

Lu, C., \& Zhang, J. (1999). Effects of water stress on photosystem II photochemistry and its thermos stability in wheat plants. Journal of Experimental Botany, 50, 1199-1206.

Lutts, S., Kinet, J.M., \& Bouharmont, J. (1996). Effects of various salts and of mannitol on ion and proline accumulation in relation to osmotic adjustment in rice (Oryza sativa L.) callus cultures. Journal of Plant Physiology, 149, 186-195.

Metcalfe, C.R. (1960). Anatomy of the monocotyledons (Vol 1). Oxford, UK: Clarendon Pr.

Nautiyal, P.C., Nageswara Rao, R.C., \& Joshi, Y.C. (2002). Moisture-deficit induced changes in leaf water content, leaf carbon exchange rate and biomass production in groundnut differing in specific leaf area. Field Crops Research, 74, 67-79.

Nawazish, S., Hameed, M., \& Naurin, S. (2006). Leaf anatomical adaptations of Cenchrus ciliaris L., from the Salt Range, Pakistan against drought stress. Pakistan Journal of Botany, 38, 1723-1730.

Ngernmuen, A. (2013). Effect of salinity on anatomical characteristics of some salt-tolerant plants in abandoned shrimp ponds (MSc Thesis). Prince of Songkla University, Songkla, Thailand.

Office of the Cane and Sugar Board. (2015). Sugarcane cultivars in Thailand. Khon Kaen, Thailand: Khon Kaen Print.

Palachai, C., Songsri, P., \& Jongrungklang, N. (2019). Comparison of yield components of Thailand. SABRAO Journal of Breeding and Genetics, 51, 80-92.

Robertson, M.J., Muchow, R.C., Donaldson, R.A., InmanBamber, N.G., \& Wood, A.W. (1999). Estimating the risk associated with drying-off strategies for irrigated sugarcane before harvest. Australian Journal of Agricultural Research, 50, 65-77. 
Santillán-Fernández, A., Santoyo-Cortés, H.V., GarcíaChávez, R.L., Covarrubias-Gutiérrez, I., \& Merino, A. (2016). Influence of drought and irrigation on sugarcane yields in different agroecoregions in Mexico. Agricultural Systems, 143, 126-135.

Shao, H.B., Chu, L.Y., Jaleel, C.A., \& Zhao, C.Z. (2008). Water-deficit stress-induced anatomical changes in higher plants. Comptes Rendus Biologies, 331, 215-225.

Searle, S.R. (1961). Phenotypic, genetic and environmental correlations. Biometrics, 17, 474-480.

Singh, K.S., Singh, P.V., Choudhury, D., Dobhal, P., Kumar, S., \& Srivastava, S. (2018). Estimation of genotypic and phenotypic correlations coefficients for yield related traits of under sodic soil. Asian Journal of Crop Science, 10, 100-106. DOI: 10.3923/ ajcs.2018.100.106

Taiz, L., \& Zeiger, E. (2002). Plant Physiology (3rd Ed.). Sunderland, Massachusetts, USA: Sinauer Associates.

Taratima, W., Ritmaha, T., Jongrungklang, N., Raso, S., \& Maneerattanarungroj, P. (2019). Anatomical Responses to Drought Stress Condition in Hybrid Sugarcane Leaf (Saccharum officinarum 'KK3'). Malaysian Applied Biology, 48, 180-188.
Udomprasert, N. (2015). Plant Stress Physiology. Bangkok, Thailand: Chulalongkorn University Press.

Van-Ittersum, M.K., \& Rabbinge, R. (1997). Concepts in production ecology for analysis and quantification of agricultural input-output combinations. Field Crop Research, 52, 197-208.

Vargas, L., Santa Brígida, A.B., Mota Filho, J.P., de Carvalho, T.G., Rojas, C.A., Vaneechouttle, D., Van Bel, M., Farrinelli, L., Ferreira, P.C., Vandepoele, K., \& Hemerly, A.S. (2014). Drought Tolerance Conferred to Sugarcane by Association with Gluconacetobacter diazotrophicus: A Transcriptomic View of Hormone Pathways. PLoS ONE, 9(12), e114744.

Wiedenfeld, R.P. (2000). Water stress during different sugar cane growth periods on yield and response to $\mathrm{N}$ fertilization. Agricultural Water Management, 43, 173-182.

Wu, L.L., Liu, Z.L., Wang, J.M., Zhou, C.Y., \& Chen, K.M. (2011). Morphological, anatomical, and physiological characteristics involved in development of the large culm trait in rice. Australian Journal of Agricultural Research, 5, 1356-1363.

Zhang, F., Zhang. K., Du, C., Li, J., Xing, Y.X., Yang, L., \& Li, Y.L. (2015). Effect of Drought Stress on Anatomical Structure and Chloroplast Ultrastructure in Leaves of Sugar cane. Sugar Tech, 17, 41-48. 